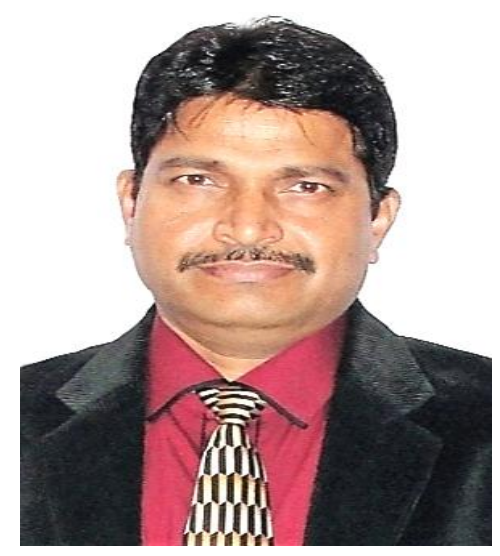

\title{
Retinopathy of prematurity: An update
}

Dear Friends

Season's Greetings!!

Retinopathy of prematurity (ROP) remains one of the leading causes of visual loss in young children. It was first described in 1942 by Dr. Theodore Terry. Infants born under 32 weeks gestation with weight $<1500$ gms (the lower the birth weight the higher the incidence) and those given high levels of supplemental oxygen are particularly at risk. In ROP there is uncontrolled neovascular process in which retinal endothelial cells stop developing and die, then later, proliferate, migrate, organize, scar and cause a detachment of the retina. It was originally called as retrolental fibroplasia. It is now known that oxygen alone is neither sufficient nor necessary to produce ROP, and no safe level of oxygen has yet been determined. Other risk factors include sepsis, anemia, Respiratory Distress Syndrome, seizures, bradycardia, apnea, mechanical ventilation, blood transfusions, intraventicular hemorrhage and multiple prenatal maternal factors like heavy smoking, diabetes, and preeclampsia.

Recent advances in the medical care of premature infants have resulted in improved survival of low birth weight babies but this has resulted in an increasing number of babies with ROP and ROP related visual loss. With this rising rate of prematurity, need for ROP screening, newer treatment option and long term follow-up.

ROP is classified into five stages: Stage 1: Demarcation line; Stage 2: Demarcation Ridge (intraretinal neovascularization); Stage 3: Extra-retinal neovascularization; Stage 4: Subtotal retinal detachment and Stage 5: Total retinal detachment. Anatomically, the area of the retina affected by ROP is divided into three zones. Plus disease is an entity with abnormal dilated vessels on the iris along with engorged and tortuous blood vessels in the retina. Additional findings may include retinal haemorrhages, poorly dilating pupil and hazy media. Pre-threshold ROP has three criteria: ROP in Zone I, any stage; Zone II with stage 2 and Plus component; Zone III or Zone II with stage 3 and Plus component but not reaching threshold clock hours. Threshold ROP is named when ROP involves Zone 1 or 2 with stage $3+$ ROP extending for 5 contiguous sectors or 8 composite sectors. Rush disease is reached when ROP involves Zone I ROP with signs of Plus disease.

The major complications of ROP include myopia, amblyopia, strabismus, glaucoma and retinal detachment during adolescence and early adulthood. Hence screening should be done of all infants of birth weight of less than $1500 \mathrm{gms}$ (though in India this is reported up to $1700 \mathrm{gms}$ ), gestational age of less than 34-35 weeks, exposed to oxygen for more than 30 days. Infants who weigh less than $1200 \mathrm{gms}$ at birth and those born at 24-30 weeks gestational age are at particularly high risk of not only developing ROP but also developing it earlier, in more aggressive forms (Rush disease). Hence smaller babies must be screened at the earliest. Other factors that can increase risk of ROP and where screening should be considered are other premature babies $(<37$ weeks and/or $<2000 \mathrm{gms})$ with Respiratory distress syndrome, sepsis, multiple blood transfusions, multiple births, apnoeic episodes and intraventricular haemorrhage. ${ }^{1}$

Ideally, one should screen all eligible babies at 31 weeks post conceptional age or 3-4 weeks after birth (whichever is earlier). Infants weighing less than 1200 grams at birth and those born at 24-30 weeks gestational age are screened early, usually not later than 2-3 weeks after birth. No examination needed in first 23 weeks of life. One screening session should be completed definitely before 'Day 30 ' of the infant's life. The recommended eye drops for dilatation include tropicamide $0.5 \%$ - $1 \%$ with phenylepherine $2.5 \%$. Two to three instillations of each of these drops, five minutes apart should be done. Cyclopentolate $0.5 \%$ to $1.0 \%$ can also be used. In case of mature retina follow-up should be done 3 monthly for 1year while in immature retina Follow-up should be done bi-weekly. In immature Zone I retina follow-up should be done weekly while in 
prethreshold ROP follow-up should be done every 3-7 days. Threshold ROP should be given early treatment within 72 hours. The goal of treatment is to destroy the retina that is deprived of retinal vessels. This helps to shrink the new vessels and prevents the formation of dense scars that usually follow.

Laser photocoagulation is the most common treatment. A laser is directed to a designated spot to ablate the avascular retina. Treatment with nearconfluent (0.5-1 burn-width) laser burn spacing should be administered to the entire avascular retina. Laser photocoagulation is the preferred method of treatment by surgeons, because there is little postoperative pain and swelling. ETROP randomized trial was done in type I ROP patients with zone I, any stage ROP with plus disease; zone I, stage 3 ROP without plus disease; zone II, stage 2 or 3 with plus disease and found reduction in unfavorable visual acuity $19.8 \%$ to $14.3 \%$ $(\mathrm{P}<.005)$ and unfavorable structural outcomes were reduced from $15.6 \%$ to $9.0 \%(\mathrm{P}<.001)$ at 9 months. $^{2}$ Type II ROP is characterized by zone I, stage 1 and 2 without plus disease and zone II, stage 3 without plus disease. Cryotherapy can be used to treat threshold ROP but is not preferred. The aim is to destroy abnormal tissue by freezing and is often used to treat Stage III ROP. Cryotherapy reduces the risk for retinal detachment from $43 \%$ to $21 \%$. $30 \%$ of treated and $51.9 \%$ of control eyes (P.001) had unfavorable structural outcomes at 15 years. $44.7 \%$ of treated and $64.3 \%$ of control eyes (P.001) had unfavorable visual acuity outcomes at 15 years. The major limitation is it causes significant swelling of the eye and eyelid, which makes postoperative assessment difficult. ${ }^{3}$

Other methods of treatment include scleral buckling with silicone band $(2.5 \mathrm{~mm}$ wide) placed circumferentially around the globe. It is used mainly for stage 4 and stage 5 retinopathies. It helps to re-attach detached retina, moves sclera (and retina) towards vitreous and changes geometry of tractional forces on retina. Greven et al found anatomic reattachment in 13 (59\%) of 22 eyes, four $(40 \%)$ of ten had $20 / 400$ or better visual acuity and follow-up of 18 months or more was done. ${ }^{4}$ Trese et al found anatomic reattachment in $12(70 \%)$ of 17 in stage $4 \mathrm{~A}$ eyes, $29(67 \%)$ of 43 in stage 4B eyes and $4(40 \%)$ of 10 in stage 5 eyes. ${ }^{5}$ Lenssparing vitrectomy has also been tried with success rate of $75 \%$ and $66 \%$ for stages $4 \mathrm{a}$ and $4 \mathrm{~b}$ respectively and $25 \%$ success rate for stage 5 ROP. ${ }^{6}$ Shah et al studied 33 eyes of 29 infants and found favorable anatomical outcome in $90 \%$ ( $9 / 10$ eyes) for stage 4A, 44.4\% (4/9 eyes) for stage $4 \mathrm{~B}$ and $14.3 \%$ (2/14) for stage 5 ROP eyes. The mean follow-up was 19.1 months. ${ }^{7}$ Gopal et al found anatomical success in $22.5 \%$ cases in stage $5 .{ }^{8}$ Anti-VEGFs have also been tried. Lalwani et al tried anti-VEGF in five eyes of three patients with progressive ROP despite peripheral laser ablation. The ROP got stabilized allowing laser supplementation.
There was varying development of tractional retinal detachments in several eyes but the ROP component quieted in all cases. ${ }^{9}$ Hugo et al investigated thirteen patients (18 eyes) with mean follow-up 6months.Neovascular regression was observed in 17 eyes. One patient with stage IV a ROP had spontaneous retinal reattachment. There were no serious ocular or systemic adverse events. ${ }^{10}$

The most effective prevention of retinopathy of prematurity is prevention of premature birth. The strict measures need to be follow by neonatal intensive care units, attending neonatologist, and pediatric ophthalmologists to track all babies with active ROP. Proper screening, early detection with appropriate treatment and regular follow-up is key step for successful management of ROP babies.

\section{References}

1. Subhadra Jalali, Raj Anand, Harsh Kumar, Mangat R Dogra, Rajvardhan Azad, FRCS (Ed); Lingam Gopal. Programme Planning and Screening Strategy in Retinopathy of Prematurity. Indian $J$ Ophthalmol. 2003;51:89-99.

2. Early Treatment for Retinopathy of Prematurity Cooperative Group Final Results of the Early Treatment for Retinopathy of Prematurity (ETROP) Randomized Trial. Trans Am Ophthalmol Soc. 2004;102:233-250.

3. Cryotherapy for Retinopathy of Prematurity Cooperative Group*:15-Year Outcomes Following Threshold Retinopathy of Prematurity. Arch Ophthalmol. 2005;123:311-9.

4. Greven C, Tasman W. Scleral buckling in stages 4B and 5 retinopathy of prematurity. Ophthalmology. 1990;97:817-820.

5. Trese MT. Scleral buckling for retinopathy of prematurity. Ophthalmology. 1994;101:23-26.

6. Young Suk Yu, Seong-Joon Kim, So Young Kim, Ho Kyung Choung, Gyu Hyung Park, Jang Won Heo. Lenssparing Vitrectomy for Stage 4 and Stage 5 Retinopathy of Prematurity. Kor J Ophthalmol. 2006;20(2).

7. PK, V Narendran, N Kalpana and KA Tawansy. Anatomical and visual outcome of stages 4 and 5 retinopathy of Prematurity. Eye. 2009;23:176-180.

8. L Gopal, T Sharma, M Shanmugam, SS Badrinath, A Sharma, SG Agraharam, A Choudhary Surgery for stage5 retinopathy of prematurity: The learning curve and evolving technique. Indian J Ophthalmol. 2000;48:101106.

9. Geeta A. Lalwani, Audina M. Berrocal, Timothy G. Murray, Maria Buch, Scott Cardone, Ditte Hess, CRA, Rose A. Johnson, RN,Carmen A. Puliafito. Off-label use of intravitreal Bevacizumab (avastin) for salvage Treatment in progressive Threshold retinopathy of Prematurity. Retina. 2008;28:S13-S18.

10. Hugo quiroz-Mercado, Maria A. Martinez-Castellanos, Myriam. Hernandez-Rojas, Hnelida Salazar-Teran, Robinson Vernon Paul Chan. Antiangiogenic therapy with Intravitreal bevacizumab for Retinopathy of prematurity. Retina. 2008;28:S19-S25.

How to cite this article: Maurya RP. Retinopathy of prematurity: An update. Ind J Clin Exp Ophthalmol. 2018;4(3):i-ii. 